\title{
Biodiversidade aquática e qualidade de rios em eucaliptais certificados no centro de Portugal
}

\author{
Aquatic biodiversity and quality of streams in certified eucalypt \\ plantations in Central Portugal
}

\section{João Manuel Oliveira' ${ }^{1 *}$, João Carlos Paulino ${ }^{2}$, Pedro Segurado ${ }^{1}$, Joana Rosado ${ }^{3}$, Rui Rivaes ${ }^{1}$, Fátima Fernandes ${ }^{1}$, Pedro Serafim ${ }^{4}$ e Maria Teresa Ferrreira ${ }^{1}$}

\begin{abstract}
Resumo
Em Portugal, mais de 150.000 ha de plantações de eucalipto (Eucalyptus globulus) são já geridas em conformidade com os sistemas internacionais de certificação. O objetivo deste trabalho foi o de avaliar a biodiversidade e a qualidade ecológica de pequenos rios associados a este tipo de povoamentos, usando diversas comunidades aquáticas - peixes, anfíbios e macroinvertebrados - bem como um índice de condição morfológica fluvial (qualidade do leito e margens). Este estudo foi conduzido em dois cursos de água localizados na zona centro de Portugal, a ribeira da Foz (média dimensão e regime hidrológico permanente) e a ribeira dos Rouxinóis (pequena dimensão e regime intermitente), com envolventes dominadas por plantações certificadas de eucalipto, e por isso com zonas ripícolas bem conservadas. A ribeira da Foz revelou associações piscícolas com elevada riqueza específica e que, embora dominadas por uma espécie exótica (Gobio lozanoi), incluíram várias espécies ameaçadas. A anfíbiofauna apresentou-se contudo pouco abundante, muito provavelmente devido à grande diversidade e densidade piscícola e à presença significativa do lagostim-vermelho-do-Louisiana (Procambarus clarkii). Neste sistema, registou-se ainda uma boa qualidade da condição morfológica, bem como uma elevada diversidade da comunidade de macroinvertebrados, reflexo de boa qualidade da água. Na ribeira dos Rouxinóis a comunidade piscícola foi próxima da potencial para este tipo de rios, e a anfíbiofauna apresentou-se diversa e abundante. No entanto, a comunidade de macroinvertebrados revelou-se muito pouco diversa, provavelmente devido à menor riqueza de habitats ou às elevadas condutividades aí verificadas. Os resultados demonstraram que rios associados a eucaliptais certificados podem apresentar boa qualidade ecológica e uma significativa biodiversidade aquática.
\end{abstract}

Palavras-chave: certificação florestal; comunidades aquáticas; Eucalyptus; sistemas fluviais; zona ripícola.

\begin{abstract}
In Portugal, more than 150.000 ha of eucalypt plantations are managed in compliance with international certification systems. The objective of this work was to evaluate the biodiversity and the ecological quality of streams associated with certified eucalypt plantations, using different aquatic communities as biological quality indicators - fish, amphibians, and macroinvertebrates - and a morphological quality index (condition of river bed and banks). This study was carried out in two streams located in the central region of Portugal, the Foz stream (medium size and permanent flow regime) and the Rouxinóis stream (small size and intermittent flow regime), surrounded by certified eucalyptus plantations and, consequently, presenting protected riparian buffer zones. The Foz stream revealed fish assemblages with a high species richness that, although dominated by an exotic taxon (Gobio lozanoi), included several endangered species. However, the amphibian fauna was not very abundant, probably due to the great diversity and density of fish and the strong presence of the red swamp crayfish (Procambarus clarkii). A good morphological condition was also found for this stream, as well as a high diversity of the macroinvertebrate community, reflecting good water quality. In the Rouxinóis stream the fish community was close to the potential for this river type and the amphibian fauna was diverse and abundant. However, a poor macroinvertebrate community was observed, most likely due to the lower habitat richness or the high water conductivity found there. The results showed that streams associated with certified eucalypt plantations can exhibit good ecological quality and a significant aquatic biodiversity.
\end{abstract}

Keywords: forest certification; aquatic communities Eucalyptus; fluvial systems; riparian zone.

1. Centro de Estudos Florestais, Instituto Superior de Agronomia, Universidade de Lisboa. Lisboa, Portugal.

2. Faculdade de Ciências, Universidade de Lisboa. Lisboa, Portugal.

3. Universidade de Évora. Évora, Portugal.

4. Altri Florestal S.A. Constância, Portugal.

* Autor correspondente: joliveira@isa.ulisboa.pt

Sci. For., Piracicaba, v. 47, n. 123, p. 383-394, set. 2019

DOI: doi.org/10.18671/scifor.v47n123.01 
Oliveira et al. - Biodiversidade aquática e qualidade de rios em eucaliptais

certificados no centro de Portugal

\section{INTRODUÇÃO}

O eucalipto (Eucalyptus globulus) foi introduzido em Portugal na segunda metade do século XIX e rapidamente se dispersou por todo o território, de tal forma que em 2013 ocupava já cerca de 812.000 ha, tornando-se a espécie florestal com maior área no país (ICNF, 2013). O eucalipto assume assim um papel importante na silvicultura em Portugal, provendo matéria-prima para a produção de pasta de papel, uma das mais importantes indústrias nacionais (BARREIRO; TOMÉ, 2012). Mais de 150.000 ha destas plantações são já certificadas, e como tal geridas de acordo com boas práticas florestais, em conformidade com os sistemas internacionais de certificação (CELPA, 2013).

Contudo, verifica-se uma particular escassez de informação científica sobre as várias comunidades aquáticas e a biodiversidade aquática em troços de rios associados a eucaliptais na Península Ibérica, nomeadamente em povoamentos certificados com zonas ripícolas protegidas. As zonas ripícolas são muito importantes para a estrutura e funcionamento dos ecossistemas aquáticos, representando diversas funcionalidades como a de "tampão térmico", fonte de invertebrados para alimento de peixes, aumento da estabilidade das margens, ou disponibilidade de abrigos lenhosos para diversas espécies, em suma, promovendo a biodiversidade (CASATTI et al., 2012; NAIMAN et al., 1993; PUSEY; ARTHINGTON, 2003; RIVAES et al., 2017; WOOTTON, 2012)

Os peixes são bons indicadores de qualidade ambiental, apresentando sensibilidade a vários níveis de perturbações biológicas, físicas e químicas (FERREIRA et al., 2007; PONT et al., 2006). Os anfíbios, por outro lado, são dependentes do meio aquático e do componente florestal terrestre; embora o tipo de habitat varie entre espécies, exibem claramente esta dupla dependência habitacional (FICETOLA et al., 2009; PAULEY et al., 2000). As comunidades de macroinvertebrados bentónicos apresentam também um papel essencial no funcionamento do meio aquático, e em pequenos rios em áreas florestadas dependem em grande parte da folhada como fonte de energia (GRAÇA et al., 2002; WOOTTON, 2012). Estas características revelam a importância destes elementos biológicos como indicadores da biodiversidade e integridade ecológica dos sistemas fluviais.

Um dos primeiros estudos sobre a diversidade piscícola e a avaliação da qualidade de rios associados a povoamentos certificados de eucalipto em zonas mediterrânicas foi realizado por Oliveira et al. (2016), na bacia do Tejo (Portugal). O presente trabalho vem dar continuidade a esse estudo, embora recorrendo a diferentes comunidades ligadas ao meio aquático, nomeadamente peixes, anfíbios e macroinvertebrados. Para tal, foram selecionados dois cursos de água associados a eucaliptais certificados no centro de Portugal e estudadas as suas comunidades biológicas. A qualidade morfológica dos locais (i.e., a maior ou menor perturbação do leito e margens) foi também quantificada.

\section{MATERIAL E MÉTODOS}

\section{Área de estudo}

Este estudo foi conduzido em dois cursos de água localizados na zona centro de Portugal: ribeira da Foz, afluente do rio Tejo (bacia hidrográfica do rio Tejo) e ribeira dos Rouxinóis, afluente do rio Rial (bacias hidrográficas das ribeiras do Oeste) (Figura 1). Típico do clima mediterrânico, a região apresenta uma forte variabilidade hidrológica natural, com maiores caudais no outono e inverno e um gradual declínio de caudal a partir do final da primavera. Os dois locais apresentam envolventes dominadas por plantações certificadas de eucalipto, exibindo por isso zonas ripícolas bem conservadas. Estas zonas de proteção semi-naturais não são utilizadas para quaisquer atividades produtivas e suportam uma vegetação ripícola pouco fragmentada, composta por vários estratos e dominada por espécies nativas. Em ambos os locais são visíveis sinais de usos agrícolas da terra no passado (antes da sua conversão para exploração florestal), incluindo a presença nas margens de alguns muros de pedra e de edificações desagregadas.

A ribeira da Foz é um sistema de pequena/média dimensão (largura média $=5 \mathrm{~m}$; profundidade média $=35 \mathrm{~cm}$ ), com regime hidrológico permanente e uma mata ripícola dominada por amieiros (Alnus glutinosa). Apresenta ainda margens pouco alteradas, uma considerável diversidade de habitats aquáticos e um substrato heterogéneo com presença razoável de elementos grosseiros (cascalho e pedras). Os parâmetros físico-químicos registados no inverno 2016 (dezembro) e primavera 2017 (março) apresentaram os seguintes valores, respetivamente: temperatura da água $=10^{\circ} \mathrm{C} ; 15^{\circ} \mathrm{C}$; condutividade $=130 \mu \mathrm{S} ; 150 \mu \mathrm{S} ; \mathrm{pH}=7,6 ; 7,6$. 


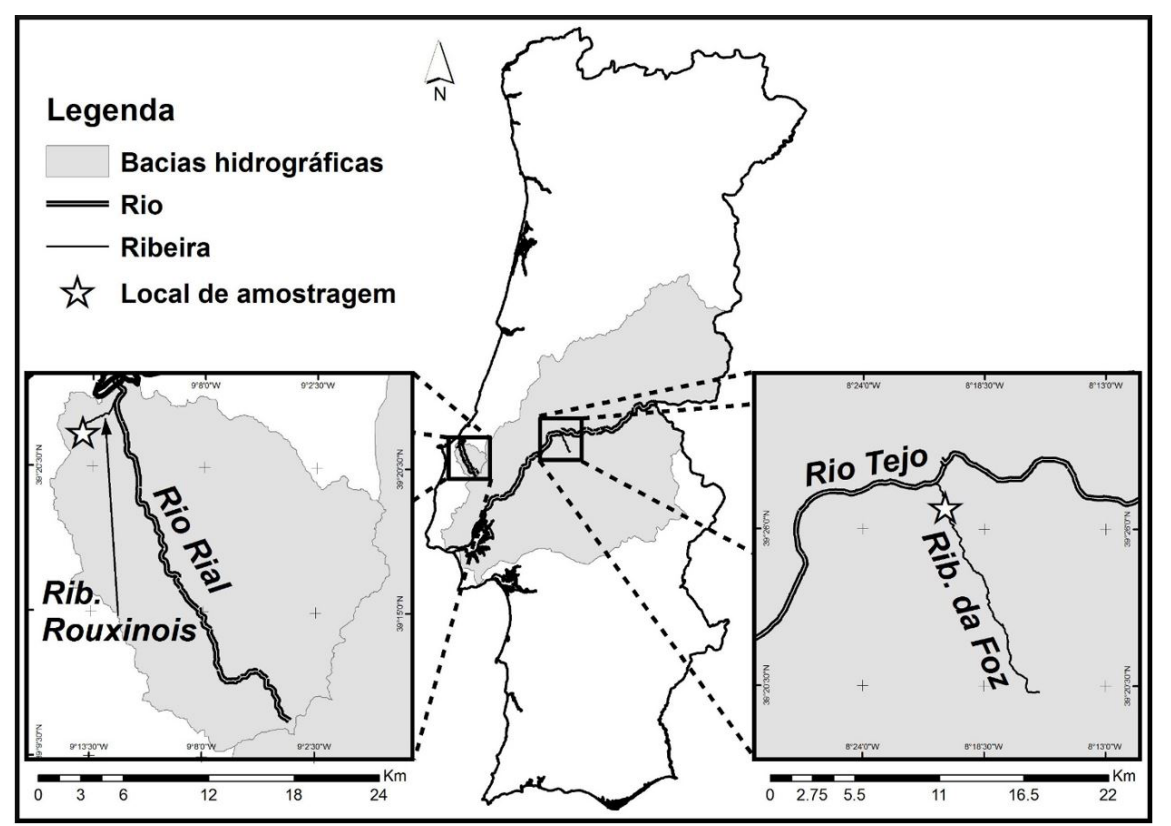

Figura 1. Mapa das áreas de estudo mostrando a localização dos dois cursos de água estudados (ribeiras da Foz e Rouxinóis).

Figure 1. Map of the study areas showing the location of the two studied streams (Foz and Rouxinóis streams).

A ribeira dos Rouxinóis apresenta ainda menor dimensão (largura média $=2 \mathrm{~m}$; profundidade média $=25 \mathrm{~cm}$ ) e um regime intermitente, de tal forma que a área molhada fica reduzida a alguns pegos mais profundos no período de estio. A mata ripícola é largamente dominada por aveleiras (Corylus avellana). Apresenta razoável diversidade de habitats aquáticos, embora as margens exibam alguma erosão e o substrato seja dominado por elementos finos e areias. Os parâmetros físico-químicos registados no inverno 2016 e primavera 2017 apresentaram os seguintes valores, respetivamente: temperatura da água $=11^{\circ} \mathrm{C} ; 15^{\circ} \mathrm{C}$; condutividade $=1250 \mu \mathrm{S} ; 1160 \mu \mathrm{S} ; \mathrm{pH}=8,2 ; 8,3$.

\section{Fauna piscícola}

Nos dois locais sob estudo amostraram-se as comunidades piscícolas em três troços fluviais distintos (montante, intermédio e jusante), em duas épocas do ano (inverno 2016 e primavera 2017). O método de captura utilizado foi a pesca elétrica, utilizando para o efeito um aparelho não portátil com output de corrente contínua (Hans Grassl EL62II). A metodologia encontra-se descrita em detalhe em Oliveira et al. (2012; 2016), salientando que as amostragens foram realizadas ao longo de troços que corresponderam a cerca de vinte vezes a largura média do rio, de forma a incluir a diversidade de habitats presentes no local (e.g., rápidos, pegos), com um comprimento mínimo de amostragem de 100 metros para garantir a representatividade da comunidade piscícola.

Para avaliar a qualidade biológica do troço jusante da ribeira dos Rouxinóis foi utilizado o índice piscícola para rios vadeáveis portugueses (F-IBIP) (INAG; AFN, 2012). O F-IBIP é um índice de integridade biótica (KARR et al., 1986) composto por um conjunto de métricas que representam aspetos da estrutura e funcionamento das ictiocomunidades. Esta ferramenta não foi contudo utilizada nos outros dois troços desta ribeira dado o baixo número de capturas, nem para os troços localizados na ribeira da Foz dados os potenciais efeitos de um grande rio adjacente (rio Tejo) nos resultados do índice (HITT; ANGERMEIER, 2006), nomeadamente ao nível da dispersão de espécies exóticas. Para apreciar a qualidade na ribeira da Foz foram por isso utilizados indicadores funcionais bem como a presença de espécies com estatuto de conservação.

Para visualizar as relações entre os locais e respetivos troços amostrados nas duas estações do ano, relativamente às suas comunidades piscícolas, foi utilizada a técnica multivariada "escalonamento multidimensional não métrico (NMDS)". Esta análise foi realizada com recurso ao software PRIMER v.6 (CLARKE; GORLEY, 2006). Tendo em vista atenuar a influência de valores extremos, transformaram-se previamente os valores das capturas por unidade de esforço $\left(500 \mathrm{~m}^{2}\right)$ utilizando a expressão $(\sqrt{\mathrm{x}})$, sendo ainda utilizada nesta análise a distância de Bray-Curtis. 
Oliveira et al. - Biodiversidade aquática e qualidade de rios em eucaliptais

certificados no centro de Portugal

\section{Anfíbiofauna}

Nas duas ribeiras sob estudo amostraram-se as comunidades de anfíbios em duas épocas do ano (inverno 2016 e primavera 2017), utilizando sempre quatro técnicas distintas em cada local: percurso pedestre com cerca de 500 metros para prospeção ativa em potenciais locais de refúgio e nas margens (RYAN et al., 2002); ponto de escuta de 10 minutos, durante o período crepuscular; arrastos com camaroeiro ao longo de uma distância aproximada de 50 metros; utilização de dez armadilhas em rede (nassas) iscadas com peixe fresco e mantidas durante cerca de seis horas na água. Em cada um dos locais foram ainda instalados quatro refúgios artificiais no solo (RYAN et al., 2002) entre os dois períodos de amostragem, sujeitos a observações quinzenais.

\section{Macroinvertebrados bentónicos}

Para cada um dos dois locais de estudo foram amostradas as comunidades de macroinvertebrados na primavera 2017. A amostragem foi realizada de acordo com a metodologia estabelecida em Portugal pela Agência Portuguesa do Ambiente (ver detalhes em INAG, 2008). Resumidamente, para cada local foi selecionado um troço de amostragem com cerca de 50 metros de extensão, onde foram efetuados seis arrastos (rede de mão; malha de $0,5 \mathrm{~mm}$ ) tendo em conta a representatividade dos habitats. A amostra para cada local incluiu as seis subamostras que foram agrupadas num mesmo recipiente. As amostras foram preservadas em formol a 4\% e em laboratório foi realizada a triagem dos indivíduos capturados e a sua identificação taxonómica.

Para avaliar a qualidade biológica com base nas comunidades de macroinvertebrados foram utilizados dois índices: Iberian Biomonitoring Working Party (IBMWP) e o Índice Português de Invertebrados do Sul ( $\left(\mathrm{IPtI}_{\mathrm{s}}\right)$. O IBMWP é um método que avalia o grau de contaminação do sistema, sendo suportado por uma tabela onde as famílias são pontuadas de acordo com a sua tolerância à contaminação das águas (ALBA-TERCEDOR et al., 2002). Por outro lado, e à semelhança do F-IBIP, o IPtI é um índice multimétrico composto por atributos de composição e abundância, que reflete a integridade das comunidades de macroinvertebrados (INAG, 2008).

\section{Condição morfológica fluvial}

Para avaliar a condição morfológica dos dois locais, isto é, a maior ou menor perturbação do leito e margens do rio, utilizou-se o índice "Grau de Qualidade do Canal" (GQC) (OLIVEIRA et al., 2007). O GQC é uma ferramenta expedita que avalia a qualidade de várias componentes associadas ao leito e margens do rio. Para o seu cálculo, em transetos regularmente distanciados, são classificadas oito variáveis físicas numa escala qualitativa (Tabela 1). Cada uma destas variáveis apresenta quatro níveis de degradação, correspondendo a pontuação mínima à situação de maior impacte. A soma das pontuações obtidas em cada uma das oito variáveis representa a qualidade física do troço.

Tabela 1. Resultados do índice Grau de Qualidade do Canal (GQC) para as ribeiras da Foz e Rouxinóis (menor pontuação dos descritores e do total implicam maior degradação).

Table 1. Results of the channel quality index (GQC) for Foz and Rouxinóis streams (lower scores of the descriptors and total imply greater degradation).

\begin{tabular}{lcc}
\hline \multicolumn{1}{c}{ Descritor } & Ribeira da Foz & Ribeira dos Rouxinóis \\
\hline Presença de estruturas de retenção (1-4) & 2 & 2 \\
Estrutura do canal (1-4) & 3 & 4 \\
Sedimentos e estabilidade do canal (1-4) & 3 & 3 \\
Condição da mata ripícola e margens (1-4) & 4 & 3 \\
Alteração artificial das margens (1-4) & 3 & 3 \\
Heterogeneidade do canal (1-4) & 3 & 3 \\
Estruturas do leito (1-8) & 6 & 3 \\
Deposição de finos intersticiais (1-4) & 3 & 2 \\
TOTAL & 27 (classe II - Bom) & 23 (classe III - Moderado) \\
\hline
\end{tabular}

\section{RESULTADOS E DISCUSSÃO}

\section{Ribeira da Foz}

A ribeira da Foz apresentou boa condição morfológica $(G Q C=27)$ e considerável heterogeneidade habitacional, bem como uma galeria ribeirinha de elevada integridade, evidenciando apenas uma ligeira perturbação do seu leito e margens (Tabela 1). Registe-se, no entanto, a perda de conetividade 
fluvial devido à presença de um açude rústico bem consolidado no limite montante do segmento estudado, que representará um obstáculo aos movimentos das espécies migradoras (FAO/DVWK, 2002).

Nos trabalhos de campo de inverno e primavera foram capturadas na ribeira da Foz sete espécies piscícolas nativas e duas espécies exóticas, representando um total de 516 indivíduos (Tabela 2). As amostragens revelaram uma considerável homogeneidade ao nível da composição piscícola, quer entre troços, quer entre as duas épocas do ano, bem expressa na ordenação NMDS (Figura 2).

Tabela 2. Número de peixes capturados nas ribeiras da Foz e Rouxinóis no inverno de 2016 e primavera de 2017; secções das ribeiras: mont. (montante); int. (intermédio); jus. (jusante); ${ }^{a}$ espécie ameaçada, ${ }^{b}$ espécie exótica.

Table 2. Number of captured fish in the Foz and Rouxinóis streams in the winter 2016 and spring 2017; stream reaches: mont. (upstream); int. (middle); jus. (downstream); ${ }^{a}$ threatened species, ${ }^{b}$ exotic species.

\begin{tabular}{|c|c|c|c|c|c|c|c|c|c|c|c|c|}
\hline \multirow{3}{*}{ Espécies } & \multicolumn{6}{|c|}{ Ribeira da Foz } & \multicolumn{6}{|c|}{ Ribeira dos Rouxinóis } \\
\hline & \multicolumn{3}{|c|}{ Inverno } & \multicolumn{3}{|c|}{ Primavera } & \multicolumn{3}{|c|}{ Inverno } & \multicolumn{3}{|c|}{ Primavera } \\
\hline & mont. & int. & jus. & mont. & int. & jus. & mont. & int. & jus. & mont. & int. & jus. \\
\hline Achondrostoma.oligolepis & 0 & 0 & 0 & 0 & 0 & 0 & 0 & 0 & 52 & 0 & 0 & 40 \\
\hline Anguilla anguilla & 1 & 2 & 1 & 4 & 2 & 3 & 2 & 5 & 0 & 1 & 1 & 1 \\
\hline Cobitis paludica & 0 & 0 & 0 & 1 & 0 & 0 & 0 & 0 & 0 & 0 & 0 & 0 \\
\hline Gobio Iozanoib & 58 & 30 & 70 & 36 & 98 & 81 & 0 & 0 & 0 & 0 & 0 & 0 \\
\hline Iberochondrostoma.Iusitanicum ${ }^{a}$ & 1 & 3 & 0 & 0 & 3 & 0 & 0 & 0 & 0 & 0 & 0 & 0 \\
\hline Lepomis gibbosus $^{b}$ & 1 & 1 & 0 & 0 & 0 & 0 & 0 & 0 & 0 & 0 & 0 & 0 \\
\hline Luciobarbus bocagei & 6 & 0 & 1 & 0 & 0 & 3 & 0 & 0 & 0 & 0 & 0 & 0 \\
\hline Petromyzon marinusa & 0 & 1 & 4 & 0 & 2 & 0 & 0 & 0 & 0 & 0 & 0 & 0 \\
\hline Pseudochondrostoma polylepis & 15 & 19 & 4 & 0 & 15 & 1 & 0 & 0 & 0 & 0 & 0 & 0 \\
\hline Squalius pyrenaicus ${ }^{a}$ & 6 & 18 & 2 & 1 & 21 & 1 & 0 & 0 & 0 & 0 & 0 & 0 \\
\hline
\end{tabular}

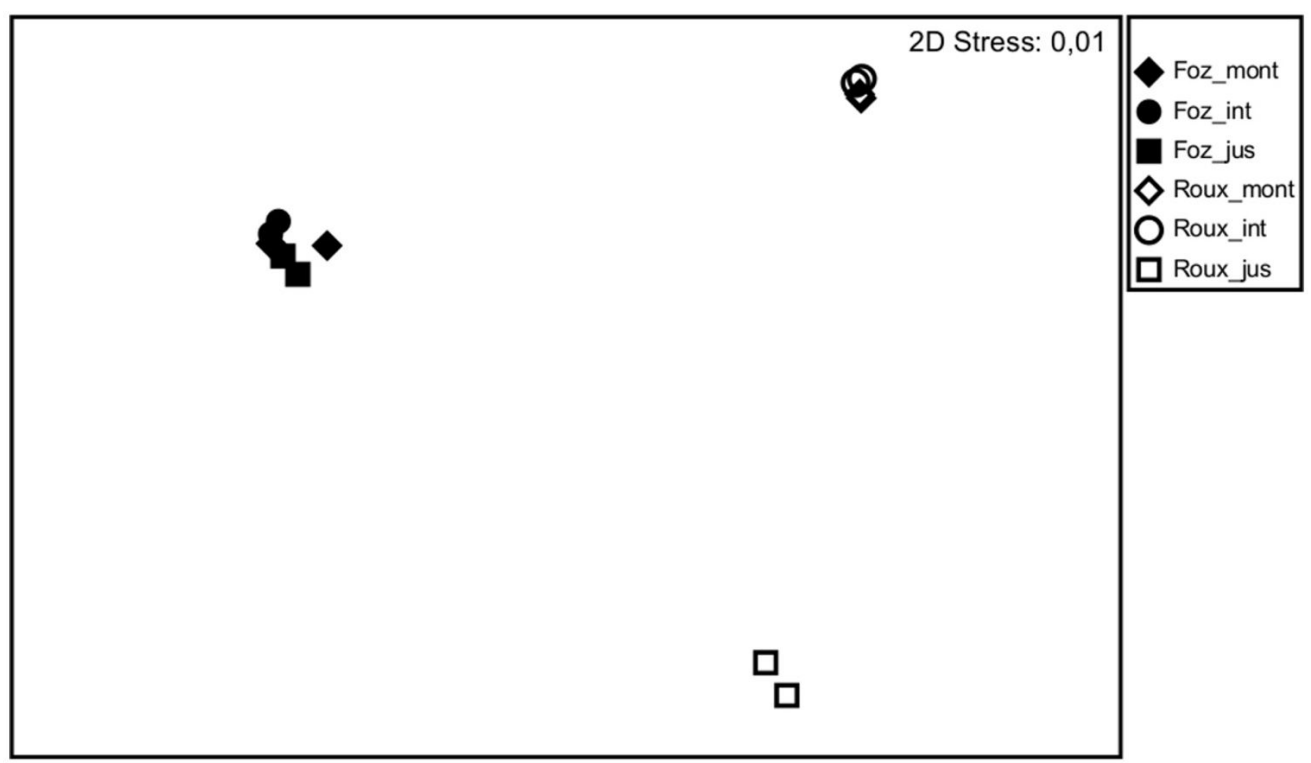

Figura 2. Gráfico do escalonamento multidimensional não métrico (NMDS) aplicado às comunidades piscícolas das ribeiras da Foz e Rouxinóis, amostradas em duas estações do ano (Matriz Bray Curtis de dados de densidade com transformação $\sqrt{\mathrm{x}}$ ); secções das ribeiras: mont (montante); int (intermédio); jus (jusante).

Figure 2. Non-metric multi-dimensional scaling (NMDS) plot of the fish assemblages for the two studied sites, which were sampled in two annual seasons (Bray Curtis matrix of $\sqrt{\mathrm{x}}$ transformed density data); stream reaches: mont (upstream); int (middle); jus (downstream).

De facto, as associações piscícolas da ribeira da Foz parecem ser significativamente dominadas pela espécie exótica Gobio lozanoi, que representou sempre mais de 40\% das capturas nos três troços fluviais em qualquer época do ano (Tabela 2). Seguiram-se em termos de abundância duas espécies nativas, Pseudochondrostoma polylepis e Squalius pyrenaicus, sobretudo bem representadas no troço intermédio. Adicionalmente a $S$. pyrenaicus, registe-se ainda a presença em ambas as amostragens de três outras espécies ameaçadas, i.e., classificadas no mínimo como vulneráveis no Livro Vermelho dos 
Vertebrados de Portugal (CABRAL et al., 2005), embora apresentando baixas densidades: Anguilla anguilla, Iberochondrostoma lusitanicum e Petromyzon marinus. É razoável crer que a elevada qualidade morfológica e da mata ripícola, bem como a boa qualidade da água da ribeira da Foz, deverão contribuir para a presença de várias espécies nativas de elevado valor conservacionista.

Por outro lado, acreditamos que a presença muito significativa de G. lozanoi na ribeira da Foz, uma espécie exótica tolerante e de grande capacidade invasora (ILHÉU et al., 2014; MUÑOZ-MAS et al., 2016), não representará um sinal de degradação do sistema, e estará sobretudo associada à sua elevada abundância no rio Tejo. De facto, este curso de água de grandes dimensões tem enorme influência na composição das comunidades piscícolas de muitos afluentes, por exemplo ao nível da presença de espécies migradoras, mas também como fonte potencial de espécies invasoras (OLIVEIRA, 2006). Estas espécies podem assim facilmente colonizar segmentos lóticos, como o do presente estudo, localizados a escassos quilómetros da confluência com o rio Tejo. Aliás, a nível trófico as associações piscícolas da ribeira da Foz são largamente dominadas por indivíduos com carácter invertívoro (incluindo G. lozanoi), reflexo da elevada integridade da cadeia alimentar do meio (KARR et al., 1986; OBERDORFF et al., 1993), como se conclui mais à frente relativamente à comunidade de macroinvertebrados. No entanto, a presença relevante da exótica G. lozanoi não deixa de representar um fator de perturbação sobre as comunidades nativas, por exemplo ao nível da competição por espaço e alimento (ALMEIDA; GROSSMAN, 2012; LEUNDA, 2010).

Relativamente à comunidade de macroinvertebrados, a ribeira da Foz apresentou uma classificação de "Bom" para ambos os índices utilizados $\left(\mathrm{IPtI}_{\mathrm{s}}=0,84\right.$ e IBMWP = 102). Os resultados refletiram a composição taxonómica observada neste sistema, onde foram amostrados um total de 355 indivíduos (ind.), distribuídos por 19 grupos taxonómicos (Tabela 3). De facto, o troço monitorizado apresentou uma comunidade muito diversa, dominada pelas famílias Leuctridae (86 ind.), Elmidae (54 ind., entre adultos e larvas) e Ephemerellidae (49 ind.), e com presença de vários taxa sensíveis à poluição orgânica das ordens Ephemeroptera, Plecoptera e Trichoptera (Tabela 3) - indicadores ecológicos de boa qualidade de água.

Tabela 3. Número de macroinvertebrados amostrados nas ribeiras da Foz e Rouxinóis e respetiva classificação taxonómica; ${ }^{a}$ adultos, ${ }^{b}$ larvas.

Table 3. Number of sampled macroinvertebrates in the Foz and Rouxinóis streams and their taxonomic classification; ${ }^{a}$ adults, ${ }^{b}$ larvae.

\begin{tabular}{|c|c|c|c|c|}
\hline Classe/ Sub classe & Ordem & Família & Ribeira da Foz & $\begin{array}{l}\text { Ribeira dos } \\
\text { Rouxinóis }\end{array}$ \\
\hline Oligochaeta & & & 37 & 16 \\
\hline Lumbriculata & Hirudinida & Erpobdellidae & 0 & 7 \\
\hline Gastropoda & Basommatophora & Ancylidae & 1 & 0 \\
\hline Gastropoda & & Lymnaeidae & 0 & 1 \\
\hline Gastropoda & & Physidae & 1 & 2 \\
\hline Gastropoda & Neotaenioglossa & Hydrobiidae & 17 & 0 \\
\hline Malacostraca & Decapoda & Cambaridae & 1 & 0 \\
\hline Insecta & Ephemeroptera & Baetidae & 19 & 0 \\
\hline Insecta & & Caenidae & 10 & 0 \\
\hline Insecta & & Ephemerellidae & 49 & 0 \\
\hline Insecta & & Leptophlebiidae & 4 & 0 \\
\hline Insecta & Plecoptera & Leuctridae & 86 & 0 \\
\hline Insecta & Trichoptera & Hydropsychidae & 8 & 0 \\
\hline Insecta & & Philopotamidae & 10 & 0 \\
\hline Insecta & Odonata & Aeshnidae & 2 & 0 \\
\hline Insecta & & Coenagrionidae & 0 & 15 \\
\hline Insecta & & Gomphidae & 27 & 0 \\
\hline Insecta & & Libellulidae & 0 & 2 \\
\hline Insecta & Coleoptera & Elmidae $^{a}$ & 1 & 0 \\
\hline Insecta & & Elmidae $^{b}$ & 53 & 0 \\
\hline Insecta & Diptera & Athericidae & 1 & 0 \\
\hline Insecta & & Chironomidae & 22 & 3 \\
\hline Insecta & & Empididae & 5 & 0 \\
\hline Insecta & & Rhagionidae & 1 & 0 \\
\hline Insecta & Hemiptera & Gerridae & 0 & 1 \\
\hline
\end{tabular}


A substituição dos povoamentos autóctones por espécies exóticas pode afetar a composição destas comunidades aquáticas, visto que a matéria orgânica produzida é naturalmente diferente da que ocorreria nas florestas nativas (por exemplo, em eucaliptais, de notar os efeitos resultantes da lixiviação de fenóis e outros compostos solúveis das folhas) (GRAÇA et al., 2002; LARRAÑAGA et al., 2009). No entanto, e à semelhança das observações de Abelho e Graça (1996) em rios do centro de Portugal, a elevada qualidade da mata ripícola na ribeira da Foz parece atenuar o impacte da monocultura de eucalipto nas comunidades de macroinvertebrados. De forma genérica, os valores elevados dos dois índices bióticos parecem assim indicar que as pressões antropogénicas, nomeadamente as associadas à silvicultura de eucalipto, não interferem significativamente na qualidade da água da ribeira da Foz.

No que se refere à anfíbiofauna foram amostradas três espécies (Tabela 4): Bufo spinosus (1 adulto) observado de forma ocasional, e ainda Salamandra salamandra (6 estados larvares) e Pelophylax perezi ( 1 adulto), ambas capturadas durante as amostragens por varrimento com camaroeiro, com valores de abundância (média e desvio padrão dos vários varrimentos realizados nas duas épocas de estudo),

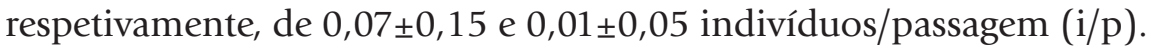

Tabela 4. Número de anfíbios amostrados nas ribeiras da Foz e Rouxinóis no inverno de 2016 e primavera de 2017. Table 4. Number of sampled amphibians in the Foz and Rouxinóis streams in the winter 2016 and spring 2017.

\begin{tabular}{|c|c|c|c|c|}
\hline \multirow{2}{*}{ Espécies } & \multicolumn{2}{|c|}{ Ribeira da Foz } & \multicolumn{2}{|c|}{ Ribeira dos Rouxinóis } \\
\hline & Inverno & Primavera & Inverno & Primavera \\
\hline Bufo spinosus & 0 & 1 & 0 & 484 \\
\hline Discoglossus galganoi & 0 & 0 & 2 & 0 \\
\hline Lissotriton boscai & 0 & 0 & 35 & 20 \\
\hline Pelophylax perezi & 0 & 1 & 0 & 1 \\
\hline Salamandra salamandra & 5 & 1 & 3 & 4 \\
\hline
\end{tabular}

A reduzida abundância de anfíbios na ribeira da Foz deve-se muito provavelmente à elevada densidade e diversidade piscícola aí registada, uma vez que os peixes afetam negativamente as populações de anfíbios por competição e/ou predação (HAYES; JENNINGS, 1986; HERWIG et al., 2013; WALSTON; MULLIN, 2007). De facto, não só a única espécie confirmada em ambiente lótico foi $P$. perezi, uma das espécies mais adaptadas à presença de peixe, como a deteção de estados larvares de $S$. salamandra apenas ocorreu em pequenos braços laterais da ribeira, de carácter lêntico e sem contacto com o curso principal durante grande parte do ano, o que limitará a presença de potenciais predadores. Esta espécie tem como habitat preferencial zonas húmidas e sombrias, nomeadamente em bosques de caducifólias nas imediações de massas de água de pequena dimensão (FERRAND DE ALMEIDA et al., 2001). É também sobejamente reconhecida a influência positiva da conservação de faixas de habitat ripícola sobre a diversidade e abundância de salamandras (KNAPP et al., 2002; VESELY; McCOMB, 2002). Relembre-se que a galeria ripícola se apresenta bem desenvolvida na ribeira da Foz, com elevado ensombramento e presença de folhada no solo. Adicionalmente, um estudo recente demonstra um efeito positivo da presença de plantações de eucalipto a larga escala sobre a probabilidade de ocorrência desta espécie na região do centro interior de Portugal (CRUZ et al., 2015). Esse facto sugere a presença de galerias ripícolas mais bem preservadas em plantações de eucaliptos do que noutros usos do solo.

A menor diversidade e sobretudo as baixas densidades de anfíbios nos vários habitats aquáticos podem também dever-se à forte presença da espécie exótica lagostim-vermelho-do-Louisiana, Procambarus clarkii, que apresentou elevada abundância $(0,82 \pm 0,61 \mathrm{i} / \mathrm{p})$. Com efeito, é comprovadamente reconhecido o impacto desta espécie sobre as populações de anfíbios, estando reportados alguns casos de colapsos populacionais após a chegada deste invasor (e.g., CRUZ et al., 2008).

\section{Ribeira dos Rouxinóis}

A ribeira dos Rouxinóis apresentou uma condição morfológica razoável (GQC = 23), i.e., abaixo de bom, resultado de alguma degradação do seu leito. Aqui inclui-se a perda de conetividade fluvial por um pequeno obstáculo entre os troços amostrados, mas sobretudo a acumulação de elementos finos e a pouca representatividade de elementos grosseiros no substrato. Cremos que a erosão moderada das margens na ribeira dos Rouxinóis, nomeadamente em zonas desprovidas de vegetação, deverá contribuir para este resultado. 
Nos trabalhos de campo de inverno e primavera foram capturadas na ribeira dos Rouxinóis duas espécies piscícolas nativas, representando um total de 102 indivíduos (Tabela 2). Tendo em conta o carácter intermitente desta ribeira e a sua pequena dimensão, já seria de esperar uma comunidade piscícola muito pouco diversa, como é típico neste tipo de sistemas mediterrânicos (ENCINA et al., 2006). A ordenação NMDS separou claramente o troço de jusante, dominado quase em absoluto por Achondrostoma oligolepis, dos troços a montante e intermédio, onde A. anguilla foi a única espécie capturada (Figura 2). Este resultado está certamente relacionado com a presença de um pequeno obstáculo entre estas duas secções, que impedirá a passagem de A. oligolepis para montante. Por outro lado, a ocorrência de A. anguilla a montante pode ser explicada pela sua grande capacidade de transpor pequenos obstáculos, incluindo movimentos por terra em solos molhados (OLIVEIRA, 2006). A presença desta espécie, embora residual, reforça a importância da gestão sustentável deste espaço, visto tratar-se de uma das espécies migradoras mais ameaçadas a nível europeu (LIMBURG; WALDMAN, 2009; SANTOS et al., 2016).

No índice F-IBIP, a ribeira dos Rouxinóis está incluída no agrupamento piscícola "Ciprinícola da Região Norte Litoral", caracterizado por associações dominadas por A. oligolepis (ver detalhes em INAG; AFN, 2012). Os valores elevados do índice para o troço jusante $(0,987$ e 0,986 nas capturas de inverno e primavera, respetivamente) seriam por isso expectáveis, indiciando uma boa qualidade biológica com base na ictiofauna.

Em termos da comunidade de macroinvertebrados, a ribeira dos Rouxinóis apresentou uma classificação de "Medíocre" para o $\operatorname{IPtI}_{\mathrm{S}}(0,26)$, e "Crítica" para o IBMWP (29). Os resultados refletiram a composição taxonómica observada neste sistema, onde apenas foram capturados 47 indivíduos, distribuídos por 8 grupos taxonómicos (Tabela 3). Desta forma, o troço monitorizado apresentou uma diversidade reduzida e uma comunidade dominada pela subclasse Oligochaeta (16 ind.) e família Coenagrionidae (15 ind.), tendo-se apenas registado a presença de grupos generalistas, capazes de habitar ecossistemas com qualidade de água variável e mais tolerantes à poluição orgânica. Não foram registados taxa mais sensíveis à degradação pertencentes às ordens Ephemeroptera, Plecoptera e Trichoptera.

A baixa diversidade de macroinvertebrados, verificada neste local, pode resultar de vários fatores, que podem até atuar de forma sinergística, como a menor variedade de habitats aquáticos, incluindo um substrato dominante de elementos finos e areias, ou as elevadas condutividades aí verificadas que limitarão o estabelecimento de muitas espécies (MERZ; CHAN, 2005; VINSON; HAWKINS, 1996). Os valores deste parâmetro deverão estar relacionados com a elevada mineralização destas águas (geologia do local dominada por rochas calcárias), embora não seja de excluir a existência de alguma poluição orgânica na ribeira dos Rouxinóis, resultante, por exemplo, de eventuais descargas difusas de origem desconhecida que contribuam para a diminuição da qualidade da água. Esta hipótese é particularmente relevante uma vez que dada a intermitência sazonal característica deste curso de água, o mesmo será mais vulnerável às pressões externas (BOULTON, 2003).

Relativamente à anfíbiofauna, foram amostradas cinco espécies (Tabela 4): P. perezi (1 adulto), Discoglossus galganoi (2 adultos), S. salamandra (7 estados larvares; 0,06 $\pm 0,13 \mathrm{i} / \mathrm{p}$ ), Lissotriton boscai

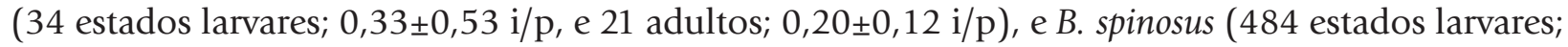
$0,26 \pm 1,11 \mathrm{i} / \mathrm{p}$ ). Para estimar a densidade de L. boscai foi aplicado o método de remoção (CARLE; STRUB, 1978) num pego isolado na primavera, tendo sido estimados 20 exemplares (18,03-21,97; intervalo de confiança a 95\%), correspondendo a uma densidade de 3,33 indivíduos $\mathrm{m}^{-2}$. Embora não fosse possível aplicar este método a B. spinosus (ausência de variação negativa consistente no número de capturas), obteve-se ainda assim uma densidade mínima de cerca de 80 indivíduos $\mathrm{m}^{-2}$. As densidades obtidas para ambas as espécies são muito elevadas. A forte presença de L. boscai nesta ribeira não é assim consistente com um estudo recente conduzido no centro interior de Portugal que sugere uma relação negativa entre a presença de plantações de eucalipto e a probabilidade de ocorrência de L. boscai (CRUZ et al., 2015).

A ribeira dos Rouxinóis revelou-se assim muito interessante do ponto de vista da herpetofauna aquática, tendo em conta o número substancial de espécies, abundâncias e densidades estimadas. Para isso contribuiu certamente a proteção da zona ripícola, uma vez que os anfíbios, durante a sua fase terrestre adulta, apresentam uma forte dependência das características dos ambientes terrestres circundantes aos meios aquáticos de reprodução (SEMLITSCH; BODIE, 2003). Como já se referiu anteriormente, veja-se por exemplo a influência positiva da conservação dos habitats ripícolas sobre a diversidade e abundância de salamandras, largamente documentada na bibliografia. No entanto, 
é muito provável que a diversidade e os elevados efetivos populacionais observados em alguns habitats lóticos estejam também relacionados com a quase ausência de peixes predadores (a espécie piscícola dominante A. oligolepis é predominantemente detritívora), ou de outros predadores como o lagostim-vermelho-do-Louisiana, muito pouco abundantes nesta ribeira.

\section{CONCLUSÕES}

Este estudo demonstra que os rios associados a eucaliptais certificados podem apresentar uma biodiversidade aquática significativa e comunidades aquáticas com relevante integridade ecológica. Revela também a multiplicidade de fatores que podem estruturar as comunidades biológicas, e as interações que estas estabelecem entre si. Veja-se o exemplo das relações bióticas que se deverão estabelecer entre anfíbios e predadores na ribeira da Foz, ou as características de habitat e qualidade da água que condicionarão as comunidades de macroinvertebrados na ribeira dos Rouxinóis. Acrescente-se ainda a dificuldade em controlar a multiplicidade de pressões que podem afetar os sistemas aquáticos, nomeadamente as "exógenas" à gestão florestal, como a colonização por espécies exóticas.

Uma gestão florestal mais sustentável deverá contribuir para a conservação dos sistemas fluviais, bem como da sua biodiversidade, embora os seus efeitos positivos representem apenas algumas das múltiplas variáveis que condicionam o funcionamento e a estrutura destes sistemas naturais.

\section{AGRADECIMENTOS}

Este trabalho foi financiado pela Altri Florestal através do projeto AquaEbio. O Centro de Estudos Florestais é uma unidade de investigação financiada pela Fundação para a Ciência e a Tecnologia I.P. (FCT), Portugal (UID/AGR/00239/2013). Rui Rivaes beneficiou de uma bolsa de Doutoramento (SFRH/BD/52515/2014) suportada pela FCT no âmbito do Programa Doutoral FLUVIO - River Restoration and Management.

\section{REFERÊNCIAS BIBLIOGRÁFICAS}

ABELHO, M.; GRAÇA, M. A. S. Effects of eucalyptus afforestation on leaf litter dynamics and macroinvertebrate community structure of streams in Central Portugal. Hydrobiologia, Dordrecht, v. 324, n. 3, p. 195-204, 1996.

ALBA-TERCEDOR, J.; JÁIMEZ-CUÉLLAR, P.; ÁLVAREZ, M.; AVILÉS, J.; BONADA, N.; CASAS, J.; MELLADO, A.; ORTEGA, M.; PARDO, I.; PRAT, N.; RIERADEVALL, M.; ROBLES, S.; SÁINZ-CANTERO, C. E.; SÁNCHEZ-ORTEGA, A.; SUÁREZ, M. L.; TORO, M.; VIDAL-ABARCA, M. R.; VIVAS, S.; ZAMORA-MUÑOZ, C. Caracterización del estado ecológico de ríos mediterráneos ibéricos mediante el índice IBMWP (antes BMWP'). Limnetica, Mislata, v. 21, n. 3-4, p. 175-185, 2002.

ALMEIDA, D.; GROSSMAN, G. D. Utility of direct observational methods for assessing competitive interactions between non-native and native freshwater fishes. Fisheries Management and Ecology, Hoboken, v. 19, n. 2, p. 157-166, 2012.

BARREIRO, S.; TOMÉ, M. Analysis of the impact of the use of eucalyptus biomass for energy on wood availability for eucalyptus forest in Portugal: a simulation study. Ecology and Society, Wolfville, v. 17, n. 2, 2012.

BOULTON, A. J. Parallels and contrasts in the effects of drought on stream macroinvertebrate assemblages. Freshwater Biology, Hoboken, v. 48, n. 7, p. 1173-1185, 2003.

CABRAL, M. J.; ALMEIDA, J.; ALMEIDA, P. R.; DELLINGER, T.; FERRAND DE ALMEIDA, N.; OLIVEIRA, M. E.; PALMEIRIM, J. M.; QUEIROZ, A. I.; ROGADO, L., SANTOS-REIS, M. Livro Vermelho dos Vertebrados de Portugal. Lisboa: Instituto da Conservação da Natureza, 2005. 659 p.

CARLE, F. L.; STRUB, M. R. A new method for estimating population size from removal data. Biometrics, Hoboken, v. 34, n. 4, p. 621-630, 1978.

CASATTI, L.; TERESA, F. B.; GONÇALVES-SOUZA, T.; BESSA, E.; MANZOTTI, A. R.; GONÇALVES, C. S.; ZENI, J. O. From forests to cattail: how does the riparian zone influence stream fish? Neotropical Ichthyology, São Paulo, v. 10, n. 1, p. 205-214, 2012. 
Oliveira et al. - Biodiversidade aquática e qualidade de rios em eucaliptais

certificados no centro de Portugal

CELPA - ASSOCIAÇÃO DA INDÚSTRIA PAPELEIRA. Boletim estatístico da indústria papeleira portuguesa. Lisboa: CELPA, 2013. 97 p.

CLARKE, K. R.; GORLEY, R. N. PRIMER V6: User Manual/Tutorial. Plymouth: PRIMER-E Ltd, 2006.190 p.

CRUZ, J.; SARMENTO, P.; CARRETERO, M. A.; WHITE, P. C. L. Exotic fish in exotic plantations: A multi-scale approach to understand amphibian occurrence in the Mediterranean region. PLoS ONE, San Francisco, v. 10, n. 6, e0129891, 2015.

CRUZ, M. J.; SEGURADO P.; SOUSA, M.; REBELO, R. Collapse of the amphibian community of the Paul do Boquilobo Natural Reserve (central Portugal) after the arrival of the exotic American crayfish Procambarus clarkii. The Herpetological Journal, London, v. 18, n. 4, p. 197-204, 2008.

ENCINA, L.; RODRÍGUEZ, A.; GRANADO-LORENCIO, C. The Iberian ichthyofauna: ecological contributions. Limnetica, Mislata, v. 25, n. 1-2, p. 349-368, 2006.

FAO/DVWK. Fish Passes - Design, Dimensions and Monitoring. Rome: FAO, 2002. 119 p.

FERRAND DE ALMEIDA, N.; FERRAND DE ALMEIDA, P.; GONÇALVES, H.; SEQUEIRA, F.; TEIXEIRA, J.; FERRAND DE ALMEIDA, F. Guias FAPAS: Anfíbios e Répteis de Portugal. Porto: FAPAS e Câmara Municipal do Porto, 2001. 249 p.

FERREIRA, T.; OLIVEIRA, J.; CAIOLA, N.; DE SOSTOA, A.; CASALS, F.; CORTES, R.; ECONOMOU, A.; ZOGARIS, S.; GARCIA-JALON, D.; ILHÉU, M.; MARTINEZ-CAPEL, F.; PONT, D.; ROGERS, C.; PRENDA, J. Ecological traits of fish assemblages from Mediterranean Europe and their responses to human disturbance. Fisheries Management and Ecology, Hoboken, v. 14, n. 6, p. 473-481, 2007.

FICETOLA, G. F.; PADOA-SCHIOPPA, E.; DE BERNARDI, F. Influence of landscape elements in riparian buffers on the conservation of semiaquatic amphibians. Conservation Biology, Hoboken, v. 23, n. 1, p. 114-123, 2009.

GRAÇA, M. A. S.; POZO, J.; CANHOTO, C.; ELOSEGI, A. Effects of Eucalyptus plantations on detritus, decomposers, and detritivores in streams. The Scientific World Journal, v. 2, p. 1173-1185, 2002.

HAYES, M. P.; JENNINGS, M. R. Decline of ranid frog species in western North America: are Bullfrogs (Rana catesbeiana) responsible? Journal of Herpetology, Saint Louis, v. 20, p. 490-509, 1986.

HERWIG, B. R.; SCHROEDER, L. W.; ZIMMER, K. D.; HANSON, M. A.; STAPLES, D. F.; WRIGHT, R. G.; YOUNK, J. A. Fish influences on amphibian presence and abundance in prairie and parkland landscapes of Minnesota, USA. Journal of Herpetology, Saint Louis, v. 47, n. 3, p. 489-497, 2013.

HITT, N. P.; ANGERMEIER, P. A. Effects of adjacent streams on local fish assemblage structure in western Virginia: implications for biomonitoring. In: HUGHES, R. M.; WANG, L.; SEELBACH, P. W. (Eds.). Landscape Influences on Stream Habitats and Biological Assemblages. Bethesda: American Fisheries Society, 2006. p. 75-86.

ICNF - INSTITUTO DA CONSERVAÇÃO DA NATUREZA E DAS FLORESTAS. 6. Inventário Florestal Nacional: Áreas dos usos do solo e das espécies florestais de Portugal continental: Resultados preliminares. Lisboa: ICNF, 2013. 34 p.

ILHÉU, M.; MATONO, P.; BERNARDO, J. M. Invasibility of Mediterranean-climate rivers by non-native fish: the importance of environmental drivers and human pressures. PloS ONE, San Francisco, v. 9, n. 11, e109694, 2014.

INAG - INSTITUTO DA ÁGUA. Manual para a avaliação biológica da qualidade da água em sistemas fluviais segundo a Directiva Quadro da Água: Protocolo de amostragem e análise para os macroinvertebrados bentónicos. Lisboa: Ministério do Ambiente, Mar, do Ordenamento do Território e do Desenvolvimento Regional, 2008.

INAG - INSTITUTO DA ÁGUA; AFN - AUTORIDADE FLORESTAL NACIONAL. Desenvolvimento de um índice de qualidade para a fauna piscícola. Lisboa: Ministério da Agricultura, Mar, Ambiente e Ordenamento do Território, 2012. 12 p.

KARR, J. R.; FAUSCH, K. D.; ANGERMEIER, P. L.; YANT, P. R.; SCHLOSSER, I. J. Assessing biological integrity in running waters: a method and its rationale. Champaign: Illinois Natural History Survey, 1986. 28 p. 
KNAPP, S. M.; HAAS, C. A.; HARPOLE, D. N.; KIRKPATRICK, R. L. Initial effects of clearcutting and alternative silvicultural practices on terrestrial salamander abundance. Conservation Biology, Hoboken, v. 17, n. 3, p. 752-762, 2002.

LARRAÑAGA, A.; BASAGUREN, A.; POZO, J. Impacts of Eucalyptus globulus plantations on physiology and population densities of invertebrates inhabiting Iberian Atlantic streams. International Review of Hydrobiology, Hoboken, v. 94, n. 4, p. 497-511, 2009.

LEUNDA, P. M. Impacts of non-native fishes on Iberian freshwater ichthyofauna: current knowledge and gaps. Aquatic Invasions, Helsinki, v. 5, n. 3, p. 239-262, 2010.

LIMBURG, K. E.; WALDMAN, J. R. Dramatic declines in North Atlantic diadromous fishes. BioScience, Oxford, v. 59, n. 11, p. 955-965. 2009.

MERZ, J. E.; CHAN, L. K. O. Effects of gravel augmentation on macroinvertebrate assemblages in a regulated California river. River Research and Applications, Hoboken, v. 21, n. 1, p. 61-74, 2005.

MUÑOZ-MAS, R.; FUKUDA, S.; VEZZA, P.; MARTÍNEZ-CAPEL, F. Comparing four methods for decision-tree induction: A case study on the invasive Iberian gudgeon (Gobio lozanoi; Doadrio and Madeira, 2004). Ecological Informatics, Amsterdam, v. 34, p. 22-34, 2016.

NAIMAN, R. J.; DECAMPS, H.; POLLOCK, M. The role of riparian corridors in maintaining regional biodiversity. Ecological Applications, Hoboken, v. 3, n. 2, p. 209-212, 1993.

OBERDORFF, T.; GUILBERT, E.; LUCCHETTA, J-C. Patterns of fish species richness in the Seine River basin, France. Hydrobiologia, Dordrecht, v. 259, n. 3, p. 157-167, 1993.

OLIVEIRA, J. M. Integridade biótica em rios ibéricos baseada em ictiotaxocenoses. 2006. 110 p. Tese (Doutorado em Engenharia Florestal) - Universidade Técnica de Lisboa, Lisboa, 2006.

OLIVEIRA, J. M.; SANTOS, J. M.; FERREIRA, M. T.; PINHEIRO, P. J.; GERALDES, A.; BOCHEICHAS, J (Coord.). Projeto AQUARIPORT: Programa Nacional de Monitorização de Recursos Piscícolas e de Avaliação da Qualidade Ecológica de Rios. Lisboa: Direção-Geral dos Recursos Florestais, 2007. 96 p.

OLIVEIRA, J. M.; FERNANDES, F.; FERREIRA, T. Effects of forest management on physical habitats and fish assemblages in Iberian eucalypt streams. Forest Ecology and Management, Hoboken, v. 363, p. 1-10, 2016.

OLIVEIRA, J. M.; SEGURADO, P.; SANTOS, J. M.; TEIXEIRA, A.; FERREIRA, M. T.; CORTES, R. V. Modelling stream-fish functional traits in reference conditions: regional and local environmental correlates. PLoS ONE, San Francisco, v. 7, n. 9, e45787, 2012.

OLIVEIRA, J. M.; SANTOS, J. M.; FERREIRA, M. T.; PINHEIRO, P. J.; GERALDES, A.; BOCHEICHAS, J (Coord.). Projeto AQUARIPORT: Programa Nacional de Monitorização de Recursos Piscícolas e de Avaliação da Qualidade Ecológica de Rios. Lisboa: Direção-Geral dos Recursos Florestais, 2007. 96 p.

PAULEY, T. K.; MITCHELL, J. C.; BUECH, R. R.; MORIARTY, J. J. Ecology and management of riparian habitats for amphibians and reptiles. In: VERRY, E. S.; HORNBECK, J. W.; DOLLOFF, C. A. (Eds.). Riparian Management in Forests of the Continental Eastern United States. Boca Raton: Lewis Publishers, 2000. p. 169-192.

PONT, D.; HUGUENY, B.; BEIER, U.; GOFFAUX, D.; MELCHER, A.; NOBLE, R.; ROGERS, C.; ROSET, N.; SCHMUTZ, S. Assessing river biotic condition at a continental scale: a European approach using functional metrics and fish assemblages. Journal of Applied Ecology, Hoboken, v. 43, n. 1, p. 70-80, 2006.

PUSEY, B. J.; ARTHINGTON, A. H.; Importance of the riparian zone to the conservation and management of freshwater fish: a review. Marine and Freshwater Research, Clayton, v. 54, n. 1, p. 1-16, 2003.

RIVAES, R.; BOAVIDA, I.; SANTOS, J. M.; PINHEIRO, A. N.; FERREIRA, T. Importance of considering riparian vegetation requirements for the long-term efficiency of environmental flows. Hydrology and Earth System Sciences Discussions, Gottingen, v. 21, p. 5763-5780, 2017.

RYAN, T. J.; PHILIPPI, T.; LEIDEN, Y. A.; DORCAS, M. E.; WIGLEY, T. B; GIBBONS J. W. Monitoring herpetofauna in a managed forest landscape: effects of habitat types and census techniques. Forest Ecology and Management, Hoboken, v. 167, n. 1, p. 83-90, 2002. 
Oliveira et al. - Biodiversidade aquática e qualidade de rios em eucaliptais certificados no centro de Portugal

SANTOS, J. M.; RIVAES, R.; OLIVEIRA, J.; FERREIRA, T. Improving yellow eel upstream movements with fish lifts. Journal of Ecohydraulics, Philadelphia, v. 1, n. 1-2, p. 50-61, 2016.

SEMLITSCH, R. D.; BODIE J. R. Biological criteria for buffer zones around wetlands and riparian habitats for amphibians and reptiles. Conservation Biology, Hoboken, v. 17, n. 5, p. 1219-1228, 2003.

VESELY, D. G.; MCCOMB, W. C. Salamander abundance and amphibian species richness in riparian buffer strips in Oregon coast range. Forest Science, Bethesda, v. 48, n. 2, p. 291-297, 2002.

VINSON, M. R.; HAWKINS, C. P. Effects of sampling area and subsampling procedure on comparisons of taxa richness among streams. Journal of the North American Benthological Society, Lawrence, v. 15, n. 3, p. 392-399, 1996.

WALSTON, L. J.; MULLIN, S. J. Responses of a pond-breeding amphibian community to the experimental removal of predatory fish. The American Midland Naturalist, Notre Dame, v. 157, n. 1, p. 63-73, 2007.

WOOTTON, J. T. River food web response to large-scale riparian zone manipulations. PLoS ONE, San Francisco v. 7, n. 12, e51839, 2012.

Recebido em: 28-09-2017

Aceito em: 26-10-2018 